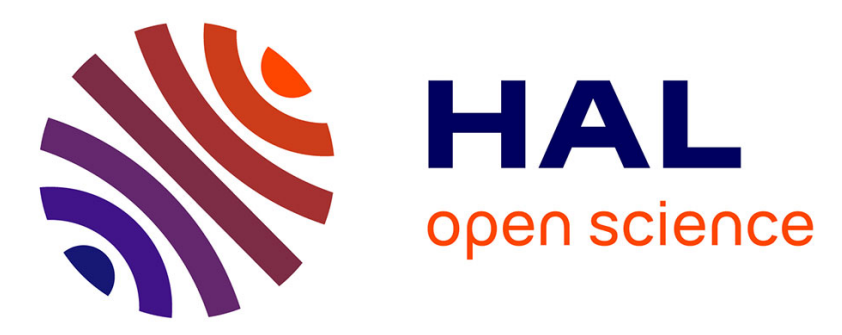

\title{
From dilute to entangled fibre suspensions involved in the flow of reinforced polymers: A unified framework
}

\author{
Marta Perez, Simon Guevelou, Emmanuelle Abisset-Chavanne, Francisco
}

Chinesta, Roland Keunings

\section{- To cite this version:}

Marta Perez, Simon Guevelou, Emmanuelle Abisset-Chavanne, Francisco Chinesta, Roland Keunings. From dilute to entangled fibre suspensions involved in the flow of reinforced polymers: A unified framework. Journal of Non-Newtonian Fluid Mechanics, 2017, 250, pp.8-17. 10.1016/j.jnnfm.2017.10.003 . hal-01671527

\section{HAL Id: hal-01671527 \\ https://hal.science/hal-01671527}

Submitted on 22 Dec 2017

HAL is a multi-disciplinary open access archive for the deposit and dissemination of scientific research documents, whether they are published or not. The documents may come from teaching and research institutions in France or abroad, or from public or private research centers.
L'archive ouverte pluridisciplinaire HAL, est destinée au dépôt et à la diffusion de documents scientifiques de niveau recherche, publiés ou non, émanant des établissements d'enseignement et de recherche français ou étrangers, des laboratoires publics ou privés. 


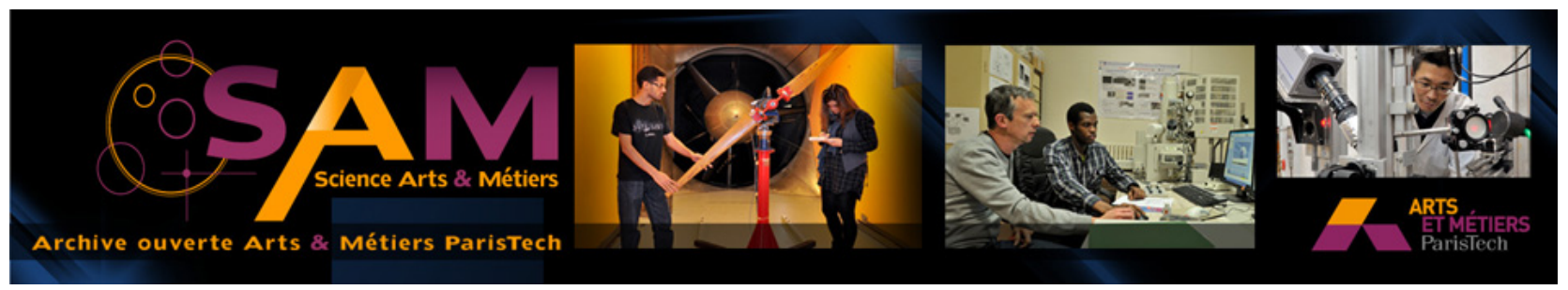

Science Arts \& Métiers (SAM)

is an open access repository that collects the work of Arts et Métiers ParisTech researchers and makes it freely available over the web where possible.

This is an author-deposited version published in: http://sam.ensam.eu

Handle ID: .http://hdl.handle.net/null

\section{To cite this version :}

M; PEREZ, S GUEVELOU, E ABISSET-CHAVANNE, F CHINESTA, R KEUNINGS - From dilute to entangled fibre suspensions involved in the flow of reinforced polymers: A unified framework Journal of Non-Newtonian Fluid Mechanics - Vol. 250, p.8-17 - 2017 


\title{
From dilute to entangled fibre suspensions involved in the flow of reinforced polymers: A unified framework
}

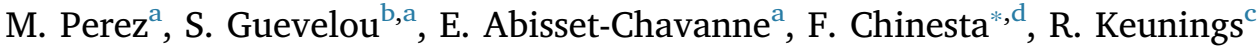 \\ ${ }^{\text {a }}$ ESI GROUP Chair \& High Performance Computing Institute, Ecole Centrale Nantes 1 rue de la (NOE), BP 92101, F-44321 Nantes cedex 3, France \\ b ESI GROUP 1 rue de la (NOE), BP 92101, F-44321 Nantes cedex 3, France \\ c ICTEAM, Université Catholique de Louvain Bat. Euler, Av. Georges Lemaitre 4, B-1348 Louvain-la-Neuve, Belgium \\ ${ }^{\mathrm{d}}$ ESI GROUP Chair \& PIMM Laboratory at ENSAM ParisTech, 151 Boulevard de l'Hopital, F-75013 Paris, France
}

Keywords:

Concentrated suspensions

Reinforced polymers

Sheet moulding compound - SMC -

Bulk moulding compound - BMC -

Entangled fibre suspensions

Multi-scale modelling

\begin{abstract}
A B S T R A C T
Most suspension descriptions nowadays employed are based on Jeffery model and some of its phenomenological adaptations that do not take into account the possible existence of a relative velocity between the fibres and the suspending fluid when the fibre interactions increase. It is expected that at very low density of contacts, as predicted by standard suspension models, fibres move with the suspending fluid velocity. When the density of fibre interactions becomes extremely high and a percolated network of fibre contacts is established within the suspension, fibres cannot move anymore and then the fluid flows throughout the rigid or moderately deformable entangled fibre skeleton, like a fluid flowing through a porous medium. In between these two limit cases, one could expect that fibres move but with a velocity lower than the one of the suspending fluid. Thus, two contributions are expected, one coming from standard suspension theory in which fibres and fluid move with the same velocity, and the other resulting in a Darcy contribution consisting of the relative fibre/fluid velocity. In this paper, we elaborate a general model able to adapt continuously to all these flow regimes.
\end{abstract}

\section{Introduction}

Over the last decades, an increasing number of functional and structural parts, made so far with metals, has been progressively reengineered by replacing metallic materials by polymers, reinforced polymers and composites. The motivation for this substitution may be the weight reduction, the simpler, cheaper or faster forming process, or the ability to exploit additional functionalities. The fillers usually employed cover a broad range involving many scales: (i) the nanometer scale (e.g. carbon nanotubes, graphene, fullerene, nanodiamonds); (ii) the micrometer to the millimeter scale (particles and short fibres); (iii) the centimeter scale of fibres used in Sheet Moulding Compound - SMC - and Bulk Moulding Compound - BMC - composite processes; and finally (iv) the macroscopic scale where fibrous reinforcements are made of continuous fibres arranged in bundles. When load-bearing capacities are especially looked for, continuous fibre reinforced polymers are selected. In that case, the impregnation of the reinforcement with a low viscosity polymer involves the flow of a Newtonian or non-Newtonian fluid in the complex multi-scale microstructure related to the fibre and tow arrangement.

Reinforced polymers are selected instead of high-performance polymers of equivalent properties since the latter are generally more expensive. When looking for functional properties, the use of nanocharges opens a wide spectrum of possibilities but also raises new challenges, such as dispersion of charges into the polymer matrix and occurrence of aggregation and disaggregation mechanisms. Suspensions of practical interest involve many scales and many concentration regimes, the latter ranging from dilute to highly concentrated.

When addressing discontinuous fibre composites, the microscopic description is usually carried out by using the unit vector $\mathbf{p}$ defining the orientation of each individual fibre. If we assume the flowing system composed of $\mathrm{F}$ fibres, then the microstructure could be described by specifying the position of each fibre centre of gravity and the associated fibre orientation, $\mathbf{X}_{i}$ and $\mathbf{p}_{i}, i=1, \cdots, \mathrm{F}$, respectively.

However, processes usually involve evolving microstructures, which means that both position and orientation evolve in time, i.e. $\mathbf{X}_{i}(t)$ and $\mathbf{p}_{i}(t)$. In the case of dilute systems, it is assumed that the fibre kinematics (displacement and rotation) do not affect any other fibre in the system.

In these circumstances, and assuming: (i) an unbounded medium with respect to the fibre size; (ii) a constant gradient of velocity of the surrounding fluid along the fibre length, (iii) the fluid Newtonian; and

\footnotetext{
* Corresponding author.

E-mail addresses: Marta.Perez-Miguel@ec-nantes.fr (M. Perez), Simon.Guevelou@esi-group.com (S. Guevelou), Emmanuelle.Abisset-Chavanne@ec-nantes.fr (E. Abisset-Chavanne), Francisco.Chinesta@ec-nantes.fr (F. Chinesta), Roland.Keunings@uclouvain.be (R. Keunings).
} 
(iv) the fibre straight and rigid enough for ignoring bending and buckling mechanisms despite the almost effective infinite aspect ratio (length to diameter ratio) and (v) the fluid velocity is unperturbed by the presence of the fibre, it was found by Jeffery almost one century ago [27] that infinite aspect ratio fibres (rods) move with the fluid, i.e. $\dot{\mathbf{X}}_{i}=\mathbf{v}\left(\mathbf{X}_{i}\right)$, with $\mathbf{v}(\mathbf{x})$ the fluid velocity at position $\mathbf{x}$, and their rotary velocity $\dot{\mathbf{p}}_{i}$ expressed by

$\dot{\mathbf{p}}_{i}=\left.\nabla \mathbf{v}\right|_{\mathbf{X}_{i}} \cdot \mathbf{p}_{i}-\left(\left.\nabla \mathbf{v}\right|_{\mathbf{X}_{i}}:\left(\mathbf{p}_{i} \otimes \mathbf{p}_{i}\right)\right) \mathbf{p}_{i}, \quad \forall i$

where $\mathbf{a}: \mathbf{b}$ refers to the tensor product of tensors $\mathbf{a}$ and $\mathbf{b}$ twice contracted.

To avoid the complexity related to the huge number of fibres involved in suspensions, the orientation distribution $\psi(\mathbf{x}, t, \mathbf{p})$ was introduced, giving the fraction of rods that at position $\mathbf{x}$ and time $t$ are oriented along direction p. Conservation of probability yields the socalled Fokker-Planck equation

$\frac{\partial \psi}{\partial t}+\nabla_{x} \cdot(\dot{\mathbf{x}} \psi)+\nabla_{p} \cdot(\dot{\mathbf{p}} \psi)=0$

where, for inertialess rods, $\dot{\mathbf{x}}=\mathbf{v}(\mathbf{x}, t)$. The Fokker-Planck equation being high-dimensional, its numerical solution using mesh-based discretization is challenging. Even if separated representations recently proposed in $[9,10,14,17]$ allow in many cases to circumvent the curse of dimensionality, coarser grained description operating at the macroscopic scale are preferred and usually considered in commercial simulation codes.

At the macroscopic scale, the orientation distribution function is substituted by its moments for describing the microstructure [4]. Usually, macroscopic descriptions of fibres suspensions are based on the use of the first two non-zero moments, i.e. the second and the fourthorder moments, a and A (odd moments vanish because $\psi(\mathbf{x}, t, \mathbf{p})=\psi(\mathbf{x}, t,-\mathbf{p}))$, defined by

$\mathbf{a}=\int_{\mathscr{S}} \mathbf{p} \otimes \mathbf{p} \psi(\mathbf{x}, t, \mathbf{p}) d \mathbf{p}$,

and

$\mathbf{A}=\int_{\mathscr{S}} \mathbf{p} \otimes \mathbf{p} \otimes \mathbf{p} \otimes \mathbf{p} \psi(\mathbf{x}, t, \mathbf{p}) d \mathbf{p}$,

where $\mathscr{S}$ is the surface of the unit ball.

The microstructural evolution described at the macroscopic scale considers the time evolution of the pdf moments. The evolution equation for the second-order orientation tensor for fibres with infinite aspect ratio (rods), is obtained by introducing expression (1) into the time derivative of Eq. (3) (see chapter 2 in [12] for additional details):

$\dot{\mathbf{a}}=\nabla \mathbf{v} \cdot \mathbf{a}+\mathbf{a} \cdot(\nabla \mathbf{v})^{T}-2 \mathbf{A}: \nabla \mathbf{v}$.

This equation involves the fourth-order moment A. The time derivative of the fourth-order moment involves the sixth-order moment $\mathscr{A}$, and so on. Thus, an approximate closure relation is needed in order to express the fourth-order moment $\mathbf{A}$ as a function of the lower-order moment a $[5,16,21,28,39]$.

In the semi-dilute regime, rod interactions cannot be neglected anymore. As interactions tend to randomize the rod orientation distribution, interactions were modeled with a diffusion term in the Fokker-Planck equation

$\frac{\partial \psi}{\partial t}+\nabla \cdot(\mathbf{v} \psi)+\nabla_{p} \cdot(\dot{\mathbf{p}} \psi)=\nabla \cdot\left(D_{r} \nabla \psi\right)$

where $\nabla$ and $\nabla_{p}$ are the gradient operator in the physical and conformational spaces respectively, and $D_{r}$ is the rotary diffusion. Thus, the microscopic and macroscopic counterparts read respectively

$\dot{\mathbf{p}}=\nabla \mathbf{v} \cdot \mathbf{p}-(\nabla \mathbf{v}:(\mathbf{p} \otimes \mathbf{p})) \mathbf{p}-D_{r} \frac{\nabla_{p} \psi}{\psi}$,

and $\dot{\mathbf{a}}=\nabla \mathbf{v} \cdot \mathbf{a}+\mathbf{a} \cdot(\nabla \mathbf{v})^{T}-2 \mathbf{A}: \mathbf{D}-6 D_{r}\left(\mathbf{a}-\frac{\mathbf{I}}{3}\right)$,

in the general 3D case (see chapter 2 in [12] for additional details).

Folgar \& Tucker [25] assumed that the rotary diffusion depends on the flow intensity through the second invariant of the rate of strain tensor, $\dot{\gamma}=\sqrt{(2 \mathbf{D}: \mathbf{D})}$,

$D_{r}=C_{I} \dot{\gamma}$,

where $C_{I}$ is the so-called interaction coefficient. Thus, the associated equation governing the evolution of the second-order orientation tensor under the Folgar \& Tucker assumption becomes

$\dot{\mathbf{a}}=\nabla \mathbf{v} \cdot \mathbf{a}+\mathbf{a} \cdot(\nabla \mathbf{v})^{T}-2 \mathbf{A}: \mathbf{D}-6 C_{I} \dot{\gamma}\left(\mathbf{a}-\frac{\mathbf{I}}{3}\right)$,

that in the sequel will be referred to as the F\&T model.

For explaining anomalous experimental orientation findings, in particular a delay in the orientation rates predicted by the F\&T model, other fibre-fibre interactions models have been proposed $[23,24,38,47,48]$. In these studies, the observed delay was attributed to the intense fibre-fibre interactions and modelled either with a modified diffusion term, a microscopic description of interactions, or by introducing a sliding mechanism between fibres and fluid. In [36], we revisited this issue and attributed the anomalous behaviour to the confinement effects that were further discussed in [35]. Obviously, there are finer approaches based on the direct simulation where the rod-rod interactions are taken into account explicitly [11,20,34].

When considering SMC processes, most of the hypotheses formulated when deriving the models just discussed become unjustifiable. Thus, in our former works we tried to analyse the effect of relaxing them all. In particular, in $[35,36,41]$ the confinement effects were widely discussed. Then, the orientation kinematics in non-Newtonian second-order fluids was addressed in [13]. The size effect that appears as soon as the flow does not exhibit a uniform strain-rate along the rod length, was deeply discussed in [3], where moreover bending mechanics were activated by the richer kinematics. Entangled systems as the ones discussed in $[32,33]$ exhibit aggregates whose kinematics was addressed in $[1,2,15]$ and chapter 2 in [12].

Compression moulding, BMC or SMC, are largely considered in the automotive industry because of its high volume capabilities. In SMC processes, a charge of a composite material, which typically consists of a matrix composed of an unsaturated polyester or vinylester, reinforced with chopped glass fibres or carbon fibre bundles and fillers, is placed on the bottom half of the preheated mould. The charge usually covers 30 to $90 \%$ of the total area. The upper half of the mould is closed rapidly at a speed of about $40 \mathrm{~mm} / \mathrm{s}$. This rapid movement causes the charge to flow inside the cavity. The reinforcing fibres are carried by the resin and experience a change of configuration during the flow. This strongly influences the mechanical properties of the final part.

The flow regime in compression moulding processes consists of a squeeze flow induced by the suspension compression. Even if the flow of a suspension does not affect "a priori" the volume fraction of fibres (except in regions in which segregation mechanisms appear), when the squeeze progresses the density of fibre interaction along the gap thickness (that continuously decreases due to the compression) increases considerably, and at the end many solid bridges across the gap thickness appear preventing the compression from progressing. Micromechanical modelling of rod contacts was addressed in a variety of works from the theoretical viewpoint $[7,19,40,42,43,45]$ being of major relevance in the direct numerical simulation of suspension flows [34].

The process simulation must track the entire fluid flow history in order to be able to predict the final reinforcement structure, and subsequently, the induced mechanical properties. Compression moulding of SMC can also generate several defects. Among them, (i) inappropriate fibre orientation with respect to the optimal one; (ii) 
segregation that results in regions in which the resin appears devoid of fibres; (iii) fibres breaking due to excessive bending generated by local flow conditions.

When analyzing the process experimentally, three different flow regimes are identified. At low density of inter-fibre contacts, and as predicted by standard suspension models, the fibres move with the suspending fluid velocity. When the density of fibre interactions becomes extremely high and a percolated network of fibre contacts is established within the suspension, the fibres cannot move anymore and then the fluid flows throughout the rigid or moderately deformable entangled fibres skeleton, like a fluid flowing through a porous medium. In between these two limit cases, one could expect that fibres move but with a velocity lower than the one of the suspending fluid, and thus two contributions are expected, one coming from standard suspension theory in which fibres and fluid move with the same velocity, and the other resulting in a Darcy contribution consisting of the relative fibre/fluid velocity. The effect of these differential velocities is observed mainly near the flow front as exposed in $[6,18]$ or in the Tjoints at the mould ribs.

Even if the two limit flow regimes just described have been extensively studied, the intermediate one, bridging both limit cases, constitutes an appealing modelling framework and whose micro-mechanical derivation constitutes the main aim of the present work.

The next section proposes a new micro-mechanical model able to account for different velocities of the fluid and reinforcement phases. Section 3 unifies the different flow regimes depending on the density of fibre interactions into a generalized Brinkman model. Because in general the addressed flows take place in narrow gaps, a lubrication approximation is proposed in Section 4, that is validated through some numerical results in Section 5.

\section{Extended micromechanical model}

We consider a fibre (of length $2 L$ ) of infinite aspect ratio, rigid enough to ignore as a first approximation bending and buckling mechanisms, and aligned in direction $\mathbf{p}$, immersed in a flow (characterized by its gradient of velocity $\nabla \mathbf{v}$ ) of a Newtonian fluid of viscosity $\eta$.

First, following the general framework proposed in our former works for addressing rods [3], aggregates [1,2], confinement [36,41] and non-Newtonian matrices [13], most of them collected in chapter 2 of [12], we proposed below a new extended model able to address the rod kinematics previously discussed. For that purpose, we consider the fibre consisting of two beads connected by a rigid connector (dumbbell model). We assume that the forces applied on each bead depend on the difference of velocities between the fluid and the bead, the first one given by $\mathbf{v}_{0}+\nabla \mathbf{v} \cdot \mathbf{p} L$ and the second one by $\mathbf{v}_{G}+\dot{\mathbf{p}} L$. Thus, the force $\mathbf{F}$ $(\mathrm{p} L)$ reads:

$\mathbf{F}(\mathbf{p} L)=\xi\left(\mathbf{v}_{0}+\nabla \mathbf{v} \cdot \mathbf{p} L-\mathbf{v}_{G}-\dot{\mathbf{p}} L\right)$,

where $\xi$ is the friction coefficient, $\mathbf{v}_{0}$ the fluid velocity at the rod centre of gravity, and $\mathbf{v}_{G}$ the velocity of the rod center of gravity.

By enforcing linear and angular momentum balances, we obtain first the velocity of the rod centre of gravity that coincides with the velocity of the fluid at that position, i.e.

$\mathbf{v}_{G}=\mathbf{v}_{0}$,

that is, the rod centre of gravity moves with the fluid, and also the expression of the rod rotary velocity $\dot{\mathbf{p}}$ (see Chapter 2 in [12]),

$\dot{\mathbf{p}}=\nabla \mathbf{v} \cdot \mathbf{p}-(\nabla \mathbf{v}:(\mathbf{p} \otimes \mathbf{p})) \mathbf{p}$,

that applies to any rod and constitutes the finest description of standard suspension kinematics.

In order to control the relative velocity between the fluid and the fibre, we consider the extended micro-mechanical model illustrated in Fig. 1 where an extra force is applied at the rod centre of gravity that scales with the relative velocity between the fibre and the mould

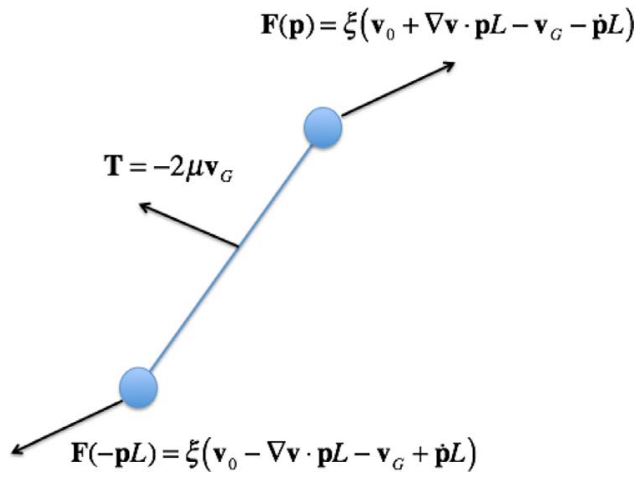

Fig. 1. Forces applied on a rod immersed in a Newtonian fluid.

velocity. The consideration of a relative velocity is needed in order to ensure the model objectivity, thus avoiding the existence of mechanical work associated with translation of the coordinate frame, that is, in order to ensure the Galilean frame-indifference. We consider the mould velocity because when the contact percolation occurs it is expected that fibres remain at rest with respect to the mould in which the flow takes place. In the sequel, we consider that the mould velocity is zero, and consequently the friction force applied at the rod center of gravity reads:

$\mathbf{T}=-2 \mu \mathbf{v}_{G}$,

where the choice of the pre-factor $2 \mu$ allows us to simplify the developments that follow.

Now, considering all forces acting on the rod: $\mathbf{F}(\mathbf{p}), \mathbf{F}(-\mathbf{p})$ and $\mathbf{T}$, the inertia-free linear momentum balance reads:

$\mathbf{0}=\mathbf{F}(\mathbf{p})+\mathbf{F}(-\mathbf{p})+\mathbf{T}=2 \xi \mathbf{v}_{0}-2(\xi+\mu) \mathbf{v}_{G}$,

from which it results

$\mathbf{v}_{G}=\frac{\xi}{\xi+\mu} \mathbf{v}_{0}$,

that proves the existence of a fibre/fluid relative velocity $\mathbf{v}_{r}$,

$\mathbf{v}_{r}=\mathbf{v}_{0}-\mathbf{v}_{G}=\frac{\mu}{\xi+\mu} \mathbf{v}_{0}$.

With $\mu \geq 0$, it results $\mathbf{v}_{G} \leq \mathbf{v}_{0}$, and we distinguish three different flow regimes:

1. Interaction-free regime, in which $\mu \approx 0$, that implies $\mathbf{v}_{G}=\mathbf{v}_{0}$.

2. Percolated case, in which $\mu \gg \xi$, that implies $\mathbf{v}_{G} \approx \mathbf{0}$.

3. Intermediate case, in which $\mathbf{v}_{G} \neq \mathbf{0}$ and $\mathbf{v}_{r} \neq \mathbf{0}$, with $\mathbf{v}_{G}+\mathbf{v}_{r}=\mathbf{v}_{0}$.

In the first tentative modelling approach proposed in this paper, we do not introduce any friction related to the rod rotary velocity. If one is interested in introducing such a rotary friction, it suffices to add a resistance torque as described in $[12,37]$ while ensuring the model objectivity. In that case, we can represent different rotary kinematics between two limit cases: (i) the one related to the standard Jeffery kinematics, and the other (ii) in which the rod rotates with the flow vorticity. In this work, we only consider the friction force, neglecting the friction torque, and then, we show that the rotary kinematics remain, without loss of generality, the one associated with the standard Jeffery model.

It is also important to note that in practical situations the gap thickness becomes smaller that the fibre length, inducing confinement and invalidating the fibre orientation kinematics discussed above. In some of our recent works $[35,36,41]$, we addressed the issue related to confinement and concluded that standard models could work quite well when using adequate empirically fitted closure relations.

In order to derive the orientation kinematics, we consider the 
balance of torques related to the mechanical system illustrated in Fig. 1. By introducing the expression of $\mathbf{v}_{G}$ given by Eq. (16) into the expression of the hydrodynamic forces $\mathbf{F}(\mathbf{p})$ and $\mathbf{F}(-\mathbf{p})$, we have

$\mathbf{F}(\mathbf{p})=\xi\left(\mathbf{v}_{r}+\nabla \mathbf{v} \cdot \mathbf{p} L-\dot{\mathbf{p}} L\right)$,

and

$\mathbf{F}(-\mathbf{p})=\xi\left(\mathbf{v}_{r}-\nabla \mathbf{v} \cdot \mathbf{p} L+\dot{\mathbf{p}} L\right)$.

Now, considering the torques with respect to the rod center of gravity, the contribution due to forces $\xi \mathbf{v}_{r}$ applied at both beads vanishes and then enforcement of a null torque results in the standard Jeffery model, whose derivation remains exactly the same as the one considered in [12], leading to Eq. (13).

Thus, from the point of view of the microstructure orientation description, there is nothing new. At the microscopic level, the rods rotary velocity is given by the standard Jeffery model

$\dot{\mathbf{p}}=\nabla \mathbf{p}-(\nabla \mathbf{v}:(\mathbf{p} \otimes \mathbf{p})) \mathbf{p}$,

leading to the mesoscopic and macroscopic models presented and discussed in Section 1.

Before finishing this section, it is important to notice that the mechanical model sketched in Fig. 1 is able to activate fibres bending. For modelling the bending mechanism, we introduce in the hydrodynamic force expression (18) the one of the rotary velocity $\dot{\mathbf{p}}(20)$, that leads to

$\mathbf{F}(\mathbf{p})=\xi \mathbf{v}_{r}+\xi(\nabla \mathbf{v}:(\mathbf{p} \otimes \mathbf{p})) \mathbf{p}$,

where the components aligned in the fibre direction does not contribute to the fibre bending. The one related to the relative velocity $\mathbf{v}_{r}$ however activates the fibre bending as soon as the relative velocity is not collinear with the fibre. Using standard results of beam theory, one can evaluate strains, stresses and bending energy in order to couple the flow analysis with failure mechanisms.

Obviously, as soon as the friction coefficient $\mu$ vanishes, the relative velocity vanishes and the standard model is recovered where fibres move with the fluid and bending is prevented because hydrodynamic forces act along the fibre direction.

The friction coefficient $\mu$ is expected to scale with the density of inter-fibre interactions, being mostly zero at the beginning of the compression process, growing when the compression progresses, and becoming very large as soon as contact percolation occurs, where it can be assumed infinite. Thus, when squeezing a suspension, different regimes can be distinguished depending on inter-fibre density of interactions. All of them are described in the next section.

\section{Throughout the different flow regimes}

As just indicated, when squeezing a suspension, five regimes can be identified: (i) the one consisting of the suspending fluid in absence of fibres associated to segregation; (ii) the one associated with negligible fibre interactions; (iii) then the one in which interaction cannot be ignored but where the fibre velocity corresponds to the one of the suspending fluid; (iv) the one in which the intense interactions induce a relative velocity between fluid and fibres; and finally (v) the very concentrated regime that results in a percolated entangled fibrous system in which the fibrous skeleton does not move anymore and the fluid flows through it. The present section covers these different flow regimes, unifying all of them in a unique Brinkman flow model, taking place in the domain occupied by the fluid at time $t, \Omega_{f}(t) \subset \Omega, \Omega$ being the whole cavity.

The most usual inertia-free flow model consists of

$\left\{\begin{array}{l}\nabla \cdot \sigma=\mathbf{0} \\ \nabla \cdot \mathbf{v}=0 \\ \sigma=-P \mathbf{I}+2 \eta \mathbf{D}+2 \eta N_{p}(\mathbf{a}: \mathbf{D}) \mathbf{a}+\beta\left(\mathbf{a}-\frac{\mathbf{I}}{3}\right) \\ \frac{D \mathbf{a}}{D t}=\nabla \mathbf{v} \cdot \mathbf{a}+\mathbf{a} \cdot(\nabla \mathbf{v})^{T}-2 \mathbf{A}^{c l}: \mathbf{D}-6 D_{r}\left(\mathbf{a}-\frac{\mathbf{I}}{3}\right)\end{array}\right.$, where $\mathbf{A}^{c l}$ refers to an appropriate closure relation expressing the fourth-order orientation tensor $\mathbf{A}$ as a function of the second-order one a, as discussed in Section 1.

In (22), different flow regimes can be distinguished:

- In absence of fibres, the above model, with $N_{p}=0$ and $\beta=0$, and ignoring the orientation equation (fourth relation in Eq. (22)), reduces to the standard Stokes problem whose solution yields the velocity, strain-rate and stress at each position $\mathbf{x} \in \Omega_{f}(t)$.

- In the case of suspensions with negligible fibre interactions the rotary diffusion vanishes, i.e. $D_{r} \approx 0$, as well as its contribution to the stress, i.e. $\beta \approx 0$. In this case, the mass and momentum equations (the first three relations in Eq. (22)) define an anisotropic Stokes flow problem for a given orientation state characterized by the microstructural descriptor $\mathbf{a}$ in the fluid domain $\Omega_{f}(t)$. It is important to note that, because inertia terms in the momentum and mass balances are neglected, the flow velocity field evolves instantaneously to adapt to the time-evolving microstructure $\mathbf{a}(x, t)$. Thus, the only characteristic time present in the model is the one related to the fibre orientation process.

- When fibre interactions cannot be ignored, all terms in the model (22) apply. Here, the F\&T assumption was employed for modelling interactions, even if as discussed in Section 1 many other, and probably better, choices exist.

In order to describe the fluid domain, we consider the fluid phase field $I(\mathbf{x}, t)$ defined as

$I(\mathbf{x}, t)=\left\{\begin{array}{ll}1 & \text { if } \mathbf{x} \in \Omega_{f}(t) \\ 0 & \text { if } \mathbf{x} \notin \Omega_{f}(t)\end{array}\right.$.

Its evolution is governed by mass conservation:

$\frac{D I}{D t}=0$,

where $\frac{D \cdot}{D t}$ is the material derivative: $\frac{D \cdot}{D t}=\frac{\partial \cdot}{\partial t}+\mathbf{v} \cdot \nabla \cdot$.

The opposite flow case is the one encountered when fibres do not move anymore and therefore the fluid flows through the porous medium associated to the entangled fibrous skeleton. This flow can be described by a standard Darcy model

$\left\{\begin{array}{l}\nabla \cdot \mathbf{v}=0 \\ \mathbf{v}=-\mathbf{K} \cdot \nabla P\end{array}\right.$

or its combined form

$\nabla \cdot(\mathbf{K} \cdot \nabla P)=0$,

again defined in $\Omega_{f}(t)$ described from $I(\mathbf{x}, t)$.

In this model, the permeability is expected to depend on the rod orientation distribution, i.e. $\mathbf{K}(\psi(\mathbf{p}))$. As discussed in the Introduction, it is usual to describe the orientation state from the different moments of the orientation distribution function, in particular its second-order moment $\mathbf{a}$, its fourth-order A, etc. Thus, the simplest choice consists in assuming that the permeability tensor only depends on the secondorder orientation tensor a, which by using the Cayley-Hamilton theorem implies its dependency on $\mathbf{I}$, $\mathbf{a}$ and $\mathbf{a}^{2}$. In what follows, we denote such a simple dependency as $\mathbf{K}(\mathbf{a})$. This kind of approach was considered from a theoretical point of view in [22]. Another route consists in creating different microstructures and identify the permeability from the direct numerical simulation of the fluid flowing through them (from the relation between the flow rate and the pressure drop [26,29,46,49] or by identifying the permeability from the dissipated power as considered in $[8,30,31])$. Now, the calculated permeabilities allow one to fit the parameters of a polynomial expression assumed in the functional dependence of the permeability of the orientation, K(a) (similarly to the route considered for constructing fitted closure relations).

When considering compression moulding, the microstructure also 
evolves because of the changes in the inter-fibre distances and not only rod orientation. To address the former other microstructural descriptors, as the ones proposed in [44], should be considered. In what follows, we consider the simplest choice in which the permeability mostly depends on the mould closing stroke, or equivalently on the mould gap at time $t$, because as proved later the orientation does not evolve very much.

Remark. All the previous flow models must be solved by enforcing appropriate initial and boundary conditions.

In between the two limit flow regimes just considered, an intermediate regime where intense hydrodynamic interactions and contacts are at the origin of a relative velocity between the fibres and the suspending fluid, is expected to exist.

When intense interactions occur, we can assume that the flow consist of one contribution coming from the suspension flow with a velocity that coincides with the one of the fibres, and another in which the fibres are assumed at rest and the fluid flows through the fibrous skeleton with the fluid/fibres relative velocity. The fluid velocity is denoted by $\mathbf{v}$, the one of the fibres that defines the suspension velocity by $\mathbf{v}^{s}$, and the relative velocity of the fluid with respect to the fibres by $\mathbf{v}^{d}$ (the Darcy contribution, assumed to be the spatial average of the actual fluid velocity). As discussed in Section 2, we have the equalities:

$\left\{\begin{array}{l}\mathbf{v}=\mathbf{v}^{s}+\mathbf{v}^{d} \\ \mathbf{v}^{s}=\frac{\xi}{\xi+\mu} \mathbf{v}=\alpha \mathbf{v} \\ \mathbf{v}^{d}=\frac{\mu}{\xi+\mu} \mathbf{v}=(1-\alpha) \mathbf{v}\end{array}\right.$.

Now, we consider the pressure drop related to both flow contributions, the one of the suspension flowing at velocity $\mathbf{v}^{s}$ (neglecting the interactions contribution to the stress, i.e. taking $\beta=0$ and assuming a quadratic closure relation),

$\nabla P^{s}=\eta \Delta \mathbf{v}^{s}+2 \eta N_{p} \nabla \cdot\left(\left(\mathbf{D}^{s}: \mathbf{a}\right) \mathbf{a}\right)$,

then the one related to the Darcy contribution,

$\nabla P^{d}=-\mathbf{K}^{-1} \cdot \mathbf{v}^{d}$,

where the permeability tensor takes into account the fact that $\mathbf{v}^{d}$ is the spatial average of the fluid velocity.

Adding both pressure drops for obtaining the total one,

$\nabla P=\nabla P^{s}+\nabla P^{d}=\eta \Delta \mathbf{v}^{s}+2 \eta N_{p} \nabla \cdot\left(\left(\mathbf{D}^{s}: \mathbf{a}\right) \mathbf{a}\right)-\mathbf{K}^{-1} \cdot \mathbf{v}^{d}$,

and writing both velocities $\mathbf{v}^{s}$ and $\mathbf{v}^{d}$ as a function of the total fluid velocity $\mathbf{v}$ according to Eq. (27), we obtain

$\nabla P=\alpha\left\{\eta \Delta \mathbf{v}+2 \eta N_{p} \nabla \cdot((\mathbf{D}: \mathbf{a}) \mathbf{a})\right\}-(1-\alpha)\left\{\mathbf{K}^{-1} \cdot \mathbf{v}\right\}$.

This has the form of the Brinkman model where the viscous and Darcy contributions are weighted by $\alpha$ and $(1-\alpha)$ respectively, with $\alpha$ depending on the friction coefficient $\mu$ that depends in turn on the density of fibre interactions.

It is important to note that this linear dependence with respect to the parameter $\alpha$ is not a modelling hypothesis, but it rather naturally derives from the proposed micromechanical modelling approach.

The solution of the extended Brinkman problem (31) yields the fluid velocity $\mathbf{v}$ and the associated suspension velocity $\mathbf{v}^{s}=\alpha \mathbf{v}$, the former allowing us to update the fluid presence function $I(\mathbf{x}, t)$ whereas the latter serves for updating the suspension presence function $I^{s}(\mathbf{x}, t)$, both evolving according to

$\frac{\partial I}{\partial t}+\mathbf{v} \cdot \nabla I=0$

and

$\frac{\partial I^{s}}{\partial t}+\mathbf{v}^{s} \cdot \nabla I^{s}=0$

respectively.

\section{Lubrication approximation}

In the case of narrow gaps, lubrication theory implies a computationally valuable dimensionality reduction. First, we consider the viscous contribution, where for the sake of simplicity we ignored the anisotropic term, i.e. we assume $N_{p}=0$ (even if it could be retained). We have

$\nabla P^{s}=\eta \Delta \mathbf{v}^{s}$,

with $\mathbf{v}^{s}=\left(u^{s}, v^{s}, w^{s}\right)^{T}$. Lubrication approximation states that the velocity gradients in the thickness direction are much larger than in-plane gradients, and that the through-the-thickness velocity component can be neglected with respect to the in-plane components:

$\left\{\begin{array}{l}\left|\frac{\partial u^{s}}{\partial x}\right| \ll\left|\frac{\partial u^{s}}{\partial z}\right| \\ \left|\frac{\partial u^{s}}{\partial y}\right| \ll\left|\frac{\partial u^{s}}{\partial z}\right| \\ \left|\frac{\partial v^{s}}{\partial x}\right| \ll\left|\frac{\partial v^{s}}{\partial z}\right| \\ \left|\frac{\partial v^{s}}{\partial y}\right| \ll\left|\frac{\partial v^{s}}{\partial z}\right| \\ w^{s} \approx 0\end{array} \mid\right.$

The last approximation in (35) leads to $P=P(x, y)$, that allows us, by integrating twice the first two equations in (34) and enforcing no-slip boundary conditions at both gap walls, to obtain:

$\mathbf{v}^{s}(x, y, z)=\frac{1}{2 \eta} \nabla P^{s}\left(z^{2}-h^{2}\right)$,

where the gap thickness is $2 h$.

The averaged velocity across the gap thickness reads

$\widetilde{\mathbf{v}}^{s}(x, y)=\frac{1}{2 h} \int_{-h}^{+h} \mathbf{v}(x, y) d z=-\frac{h^{2}}{3 \eta} \nabla P^{s}$,

where the out-of-plane component almost vanishes, i.e. $w^{s} \approx 0$. The previous equation can be rewritten as

$\nabla P^{s}=-\frac{3 \eta}{h^{2}} \widetilde{\mathbf{v}}^{s}$

The Darcy contribution, assuming that the out-of-plane velocity also vanishes, reads

$\nabla P^{d}=-\mathbf{K}^{-1} \cdot \mathbf{v}^{d}$

where $\mathbf{v}^{d}$ is constant across the narrow gap and then coincides with its averaged value $\mathbf{v}^{d}=\widetilde{\mathbf{v}}^{d}$, leading to

$\nabla P^{d}=-\mathbf{K}^{-1} \cdot \widetilde{\mathbf{v}}^{d}$.

Now, adding both pressure contributions, we obtain

$\nabla P=-\frac{3 \eta}{h^{2}} \widetilde{\mathbf{v}}^{s}-\mathbf{K}^{-1} \cdot \widetilde{\mathbf{v}}^{d}$,

and by expressing both velocities from the one related to the fluid, the pressure gradient finally reads

$\nabla P=-\alpha \frac{3 \eta}{h^{2}} \widetilde{\mathbf{v}}-(1-\alpha) \mathbf{K}^{-1} \cdot \widetilde{\mathbf{v}}$.

The following two limit cases can be identified:

- Newtonian fluid flow. In this case $\alpha=1$, and we obtain the standard lubrication counterpart of the Stokes model. By enforcing mass conservation, we obtain the boundary value problem - BVP - that allows us to calculate the pressure field and from it the flow kinematics.

- Fluid flowing through a fibrous medium at rest. In that case $\alpha=0$, and the previous equation reduces to the standard Darcy model with the 
permeability depending on the microstructure from the secondorder orientation tensor. Again, by enforcing mass conservation, we obtain the BVP governing the pressure distribution and from it the fluid phase velocity.

For intermediate values of $\alpha$, we can define an effective permeability:

$\widetilde{\mathbf{K}}^{-1}=\alpha \frac{3 \eta}{h^{2}} \mathbf{I}+(1-\alpha) \mathbf{K}^{-1}$.

This allows us to define the effective Darcy flow model

$\nabla P=-\widetilde{\mathbf{K}}^{-1} \cdot \widetilde{\mathbf{v}}$,

or

$\widetilde{\mathbf{v}}=-\widetilde{\mathbf{K}} \cdot \nabla P$

\subsection{Squeeze flow in narrow gaps}

When squeezing the fluid in a narrow gap of thickness $2 h$ with a compression rate $\mathscr{U}$, the flow-rate $\mathbf{q}(x, y)$ is given by

$\mathbf{q}(x, y)=2 h \widetilde{\mathbf{v}}(x, y)$,

from which the mass conservation reads

$\nabla \cdot \mathbf{q}=\mathscr{U}$.

Now, by replacing expression (46) into Eq. (47), we have

$-2 h \nabla \cdot(\widetilde{\mathbf{K}} \cdot \nabla P)=\mathscr{U}$,

or

$\nabla \cdot(\widetilde{\mathbf{K}} \cdot \nabla P)=-\widetilde{\mathscr{U}}$

with $\widetilde{\mathscr{U}}=\frac{\mathscr{l}}{2 h}$.

It is always possible to derive richer lubrication models, incorporating anisotropy terms of the suspension model as well as considering more complex contact conditions between suspension and gap wall, as the one considering a lubricated sliding.

\section{Numerical results}

In this section, we consider two squeezing scenarios, the first one concerning the compression of a thin disc that can be solved analytically, and the second one involving a more complex geometry.

\subsection{Compression of a thin disc}

We consider a disc of initial radius and height $r_{0}$ and $h_{0}$ respectively, with $h_{0} \ll r_{0}$. The disc is compressed at rate $\mathscr{U}$. Mass conservation implies volume conservation:

$\pi r_{t}^{2} h=\pi r_{0}^{2} h_{0}$

where $r_{t}$ and $h$ are respectively the disc radius and height at time $t$. Eq. (50) leads to

$r_{t}=\sqrt{\frac{h_{0}}{h}} r_{0}$

where the disc thickness at time $t$ is given by

$h=h_{0}-\mathscr{U} t$

The flow radial velocity $\widetilde{v}_{r}$ comes again from mass conservation,

$\pi r_{t}^{2} \mathscr{U}=2 \pi r_{t} h \widetilde{v}_{r}\left(r=r_{t}, t\right)$

or

$\widetilde{v}_{r}\left(r=r_{t}, t\right)=\frac{1}{2} \frac{r_{t}}{h} \mathscr{U}$,
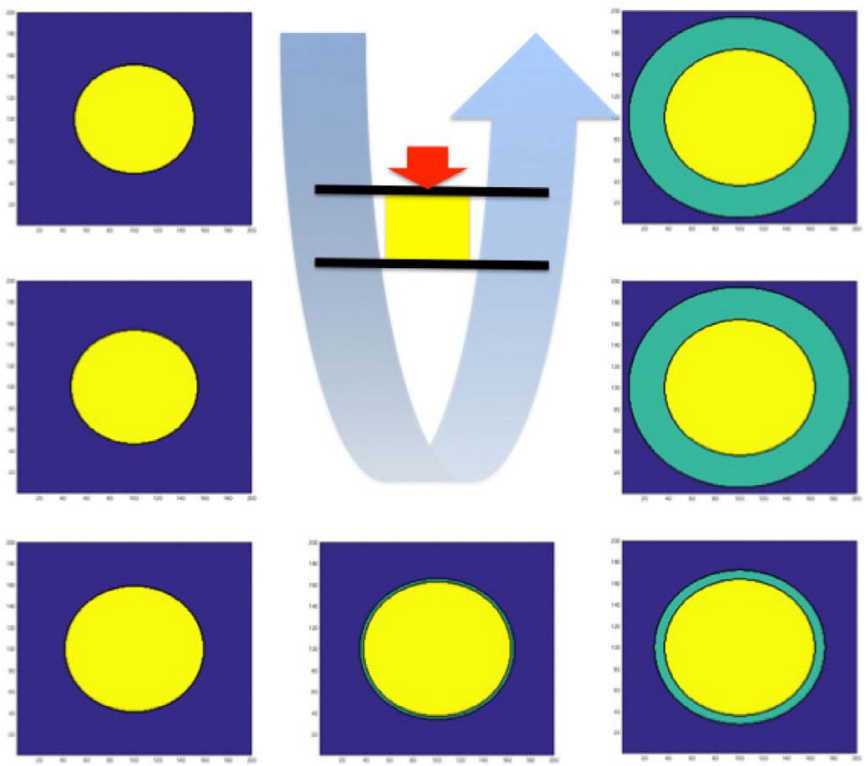

Fig. 2. Evaluating suspension segregation.

with $h$ expressed from Eq. (52). In this example, we consider a perfect sliding at both plate surfaces.

The suspension radial velocity is expressed from

$\widetilde{v}_{r}^{s}\left(r=r_{t}, t\right)=\alpha \widetilde{v}_{r}\left(r=r_{t}, t\right)$,

that allows us to describe the segregation process once the coefficient $\alpha$ is adequately expressed. In this first academic example, we consider the following evolution:

$\alpha(h)= \begin{cases}1 & \text { if } h \geq \frac{3}{4} h_{0} \\ 4 \frac{h}{h_{0}}-2 & \text { if } \frac{3}{4} h_{0} \geq h \geq \frac{1}{2} h_{0}, \\ 0 & \text { if } \frac{1}{2} h_{0} \geq h\end{cases}$

and the compression progresses until reducing the disc height by half, i.e. while $h \geq \frac{h_{0}}{2}$.

Fig. 2 depicts the evolution of the domain occupied by the suspension (yellow region) and the one occupied by the resin that segregates from the suspension due to the fibre/fluid relative velocity (green area).

The orientation process deserves a detailed analysis. At time $t$, the radial component of the flow velocity (the circumferential component vanishes because the flow problem symmetry) reads

$\tilde{v}_{r}^{s}(r, t)=\frac{\mathscr{U}}{2 h} r$,

which gives the associated cartesian components

$\left\{\begin{array}{l}\widetilde{u}^{s}(x, y, t)=\frac{\mathscr{H}}{2 h} x=\beta x \\ \widetilde{v}^{s}(x, y, t)=\frac{\mathscr{H}}{2 h} y=\beta y\end{array}\right.$,

from which we can obtain the plane component of the velocity gradient:

$\mathscr{P}\left(\nabla \widetilde{\mathbf{v}}^{s}\right)=\left(\begin{array}{ll}\beta & 0 \\ 0 & \beta\end{array}\right)$,

where $\mathscr{P}(\bullet)$ is the plane component of tensor $(\bullet)$. The flow incompressibility is not compromised because the null trace of the total velocity gradient is ensured by the through-the-thickness velocity gradient, that is by the squeezing flow.

Assuming a test rod with planar orientation, i.e. $\mathbf{p}^{T}=(\cos \theta, \sin \theta, 0)$, using the Jeffery equation we obtain a null rotary 

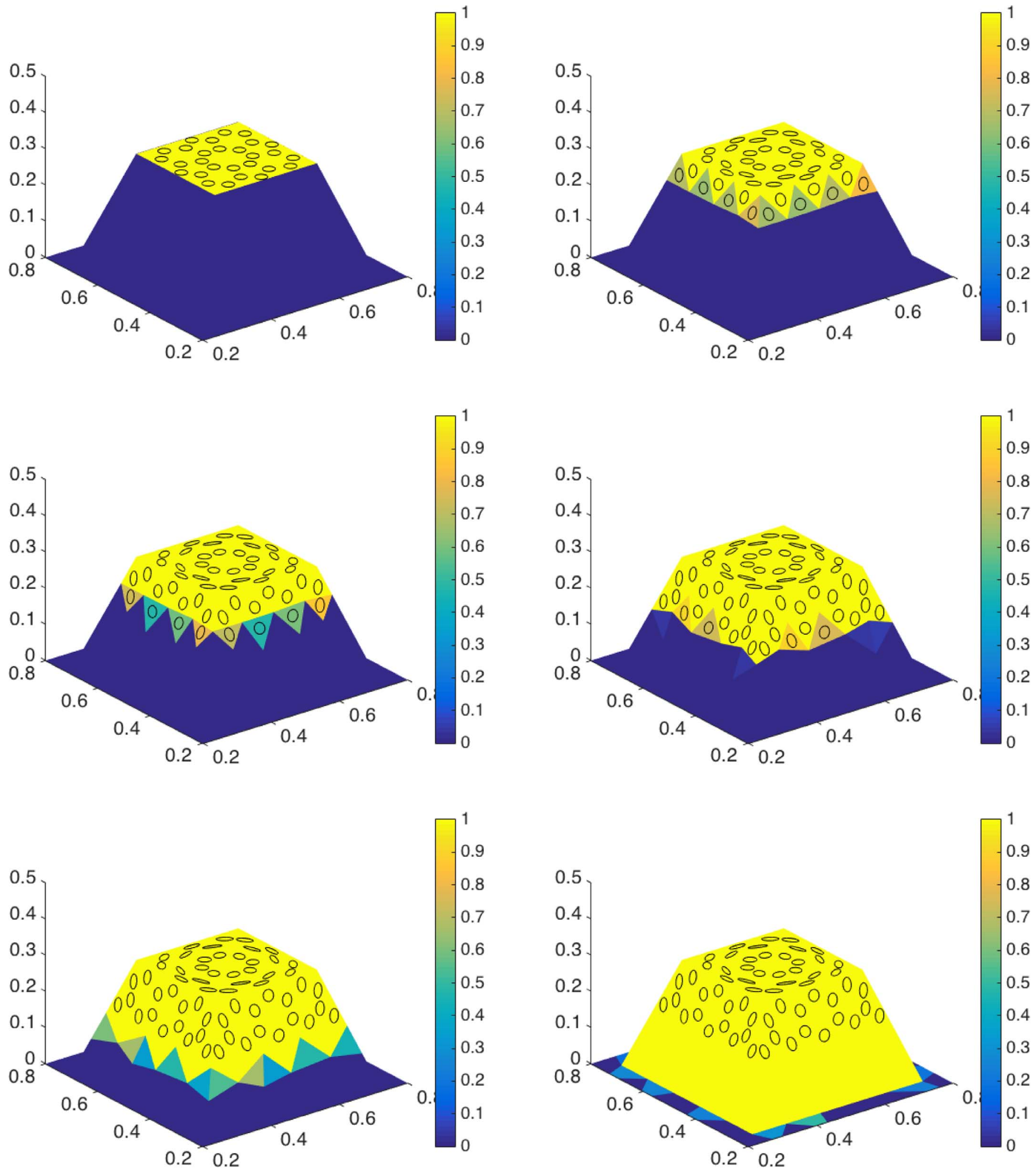

Fig. 3. Filling snapshots of a part (reinforced fluid initially located on the top surface): simulation with the generalized model.

velocity, that is $\dot{\theta}=0$. This results seems a bit conter-intuitive and deserves a discussion. If rods are assumed to have a planar orientation (i.e. their out-of-plane component vanishes), the rod kinematics is determined by the planar component of the velocity gradient. If we assume the general form of the planar component of the velocity gradient given by
$\mathscr{P}(\nabla \mathbf{v})=\left(\begin{array}{ll}G_{x x} & G_{x y} \\ G_{y x} & G_{y y}\end{array}\right)$,

Jeffery's equation (1), with $\mathbf{p}=(\cos \theta, \sin \theta)^{T}$ and consequently $\dot{\mathbf{p}}=\dot{\theta}(-\sin \theta, \cos \theta)^{T}$ reads 

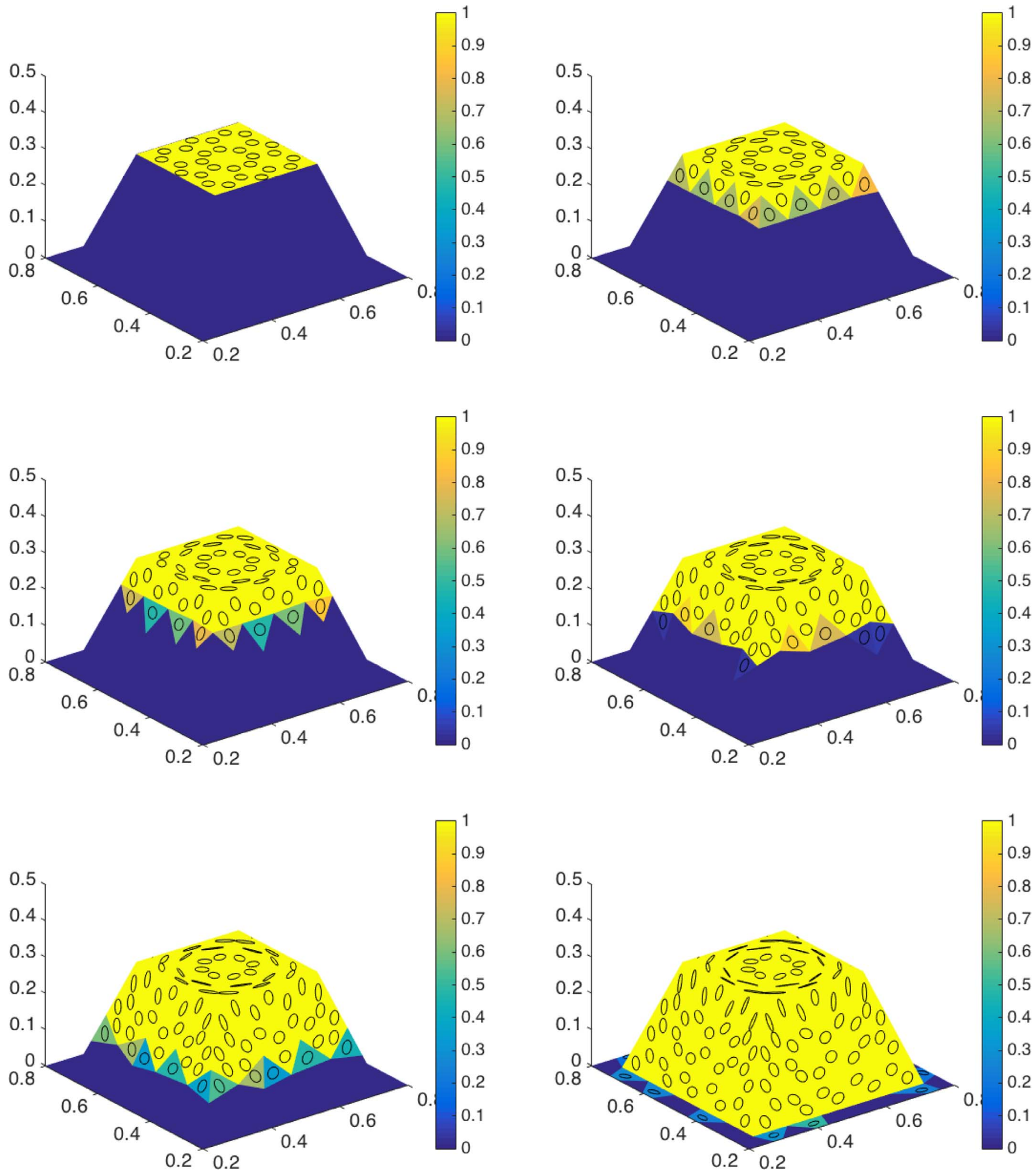

Fig. 4. Filling snapshots of a part (reinforced fluid initially located on the top surface): simulation without accounting for segregation phenomena.

$\dot{\theta}\left(\begin{array}{c}-\sin \theta \\ \cos \theta\end{array}\right)=\left(\begin{array}{ll}G_{x x} & G_{x y} \\ G_{y x} & G_{y y}\end{array}\right)\left(\begin{array}{c}\cos \theta \\ \sin \theta\end{array}\right)+\mathrm{T}\left(\begin{array}{c}\cos \theta \\ \sin \theta\end{array}\right)$,

where $\mathrm{T}$ is the scalar $\nabla \mathbf{v}$ : $(\mathbf{p} \otimes \mathbf{p})$. Now, multiplying the first row by $-\sin \theta$, the second by $\cos \theta$, and adding both of them, we obtain

$\dot{\theta}=-G_{x x} \sin \theta \cos \theta-G_{x y} \sin ^{2} \theta+G_{y x} \cos ^{2} \theta+G_{y y} \sin \theta \cos \theta$.
In the case considered above, with $G_{x x}=G_{y y}$ and $G_{x y}=G_{y x}=0$, we get $\dot{\theta}=0$ as announced, however, in the case of a radial diverging or converging flow, $\dot{\theta} \neq 0$, thus inducing respectively an alignment along the circumferential and radial directions.

Thus, when squeezing a disc the fibre orientation does not change. The microstructure, almost planar from the very beginning, does not evolve significantly and so, the mould filling process has to be 
simulated only for anticipating segregation issues.

\subsection{Numerical simulation}

In this section, we consider a more complex geometry, a sort of a rectangular hat, where the reinforced resin is initially placed on the top surface. Then compression is applied and the suspension is pushed, filling the mould. The lubrication approach is considered and the flow model adapts to the different flow regimes controlled by $\alpha$.

The flow and the associated filling and orientation fields are depicted in Fig. 3, where segregation induced by the particular choice of $\alpha(h)$ can be noticed. In this figure, the colours refer to the fluid element filling degree $(I(\mathbf{x}, t)$ in reference to Eq. (32)), thus, yellow elements are fully filled by the fluid whereas those in blue remain empty. In this figure, ellipses have been associated to all the elements fully occupied by the fibre suspension $\left(I^{s}(\mathbf{x}, t)\right.$ in reference to Eq. (33)), and additionally ellipses indicate the existing orientation. In this figure, the triangulation of the mould (fluid domain) does not correspond to the one considered for solving the flow and orientation equations. The computational mesh was coarsened in order to better represent the solution, in particular the orientation state described from an ellipse, whose axes length corresponds to the orientation tensor eigenvalues and their direction, i.e. their eigenvectors. Fig. 4 depicts the solution at the same instants for a suspension where no segregation effects are taken into account, i.e. $\alpha=1$.

The velocity field being almost governed by the compression rate and the fluid incompressibility, the time evolution of the fluid domains is almost the same when considering or not segregation mechanics. The applied pressure in both cases is different however because of the different rheologies. From the microstructural point of view, it can be noticed in Fig. 4 that at the upper surface the suspension model exhibits more intense orientations than the generalized model involving segregation. This behavior can be explained by the fact that when considering the suspension model (without segregation) the orientation mechanisms act all along the simulation, while in the other case the orientation mechanism stops as soon as the fibre contacts percolate.

If the parameter $\alpha$ depends on position, the fluid/fibre relative velocity will depend also on position, and other than predict the filling and the orientation, we could have access to the fibre concentration. It can be understood that a micro-mechanical model for parameter $\alpha$ becomes of major interest.

\section{Conclusions}

In this work, we proposed a 3D modelling framework and its lubrication counterpart able to described the flow of suspensions when considering different density of fibre interactions. A generalized model, with solid micro-mechanical foundations, allowed us to derive a Brinkman model that embraces all possible models: from the one in which no interaction occurs to the one in which segregation occurs due to too intense interactions.

Numerical results showed that the main modelling features are well recovered in the model solution, opening many valuable routes for the efficient simulation of composite processing technologies such as compression moulding or SMC.

The development of a micro mechanical model for evaluating $\alpha$, the consideration of fibre bending and buckling and the 3D solution of the flow model for addressing more complex geometries involving regions in which the lubrication approximation fails and then 3D models become compulsory, are some of the works in progress. Other important issues also considered in our works in progress concern the introduction of the fibre concentration and its associated evolution equation, the consideration of lubricated boundary conditions at the upper and bottom plates within the squeeze flow modelling framework and finally the necessity of considering fluctuations of the effective viscosity (related to fluctuations in the density of entanglements) and the evaluation
References

[1] E. Abisset-Chavanne, R. Mezher, S.L. Corre, A. Ammar, F. Chinesta, Kinetic theory microstructure modeling in concentrated suspensions, Entropy 15 (2013) 2805-2832.

[2] E. Abisset-Chavanne, F. Chinesta, J. Ferec, G. Ausias, R. Keunings, On the multiscale description of dilute suspensions of non-brownian rigid clusters composed of rods, J. Non-Newtonian Fluid Mech 222 (2015) 34-44.

[3] E. Abisset-Chavanne, J. Ferec, G. Ausias, E. Cueto, F. Chinesta, R. Keunings, A second-gradient theory of dilute suspensions of flexible rods in a newtonian fluid, Arch. Comput. Methods Eng 22 (3) (2015) 511-527.

[4] S. Advani, Ch. Tucker, The use of tensors to describe and predict fibre orientation in short fibre composites, J. Rheol. 31 (1987) 751-784.

[5] S. Advani, Ch. Tucker, Closure approximations for three-dimensional structure tensors, J. Rheol. 34 (1990) 367-386.

[6] S. Advani, K.-T. Hsiao, Edts. Manufacturing Techniques for Polymer Matrix Composites, Woodhead Publishing, 2012.

[7] A. Allaoui, S. Toll, P. Evesque, J. Bai, On the compressive response of carbon nanotube tangles, Phys. Lett. A 373 (2009) 3169-3173.

[8] A. Ammar, E. Abisset-Chavanne, F. Chinesta, R. Keunings, Flow modelling of quasinewtonian fluids in two-scale fibrous fabrics. advanced simulations, Int. J. Mater. Form. 10 (2017) 547-556.

[9] A. Ammar, B. Mokdad, F. Chinesta, R. Keunings, A new family of solvers for some classes of multidimensional partial differential equations encountered in kinetic theory modelling of complex fluids, J. Non-Newtonian Fluid Mech. 139 (2006) 153-176.

[10] A. Ammar, B. Mokdad, F. Chinesta, R. Keunings, A new family of solvers for some classes of multidimensional partial differential equations encountered in kinetic theory modelling of complex fluids. part II: transient simulation using space-time separated representations, J. Non-Newtonian Fluid Mech. 144 (2007) 98-121.

[11] G. Ausias, X.J. Fan, R. Tanner, Direct simulation for concentrated fibre suspensions in transient and steady state shear flows, J. Non-Newtonian Fluid Mech. 135 (2006) $46-57$.

[12] C. Binetruy, F. Chinesta, R. Keunings, Flows in Polymers, Reinforced Polymers and Composites, A multiscale approach, Springerbriefs, Springer, 2015.

[13] D. Borzacchiello, E. Abisset-Chavanne, F. Chinesta, R. Keunings, Orientation kinematics of short fibres in a second-order viscoelastic fluid, Rheol. Acta. 55 (2016) 397-409.

[14] F. Chinesta, A. Ammar, A. Leygue, R. Keunings, An overview of the proper generalized decomposition with applications in computational rheology, J. Non Newtonian Fluid Mech. 166 (2011) 578-592.

[15] F. Chinesta, From single-scale to two-scales kinetic theory descriptions of rods suspensions, Arch. Comput. Methods Eng. 20/1 (2013) 1-29.

[16] D.H. Chung, T.H. Won, Improved model of orthotropic closure approximation for flow induced fiber orientation, Polym. Compos. 22/5 (2001) 636-649.

[17] F. Chinesta, R. Keunings, A. Leygue, The Proper Generalized Decomposition for Advanced Numerical Simulations, A primer, Springerbriefs, Springer, 2014.

[18] S.K. De, J.R. White, Short fibre-Polymer Composites, Woodhead Publishing, 1996.

[19] M. Doi, S.F. Edwards, Dynamics of rod-like macromolecules in concentrated solution, Part 1. J. Chem. Soc., Faraday Trans. 2 (74) (1978) 560-570.

[20] P. Dumont, S.L. Corre, L. Orgeas, D. Favier, A numerical analysis of the evolution of bundle orientation in concentrated fibre-bundle suspensions, J. Non-Newtonian Fluid Mech. 160 (2009) 76-92.

[21] F. Dupret, V. Verleye, Modelling the Flow of Fibre Suspensions in Narrow Gaps, in: D. Siginer, D. De Kee, R. Chabra (Eds.), Advances in the Flow and Rheology of NonNewtonian Fluids, Rheology Series, Elsevier, 1999, pp. 1347-1398.

[22] S. Federico, W. Herzog, On the permeability of fibre-reinforced porous materials, Int. J. Solids Struct. 45 (2008) 2160-2172.

[23] J. Ferec, G. Ausias, M.-C. Heuze, P. Carreau, Modeling fiber interactions in semi concentrated fiber suspensions, J. Rheol. 53/1 (2009) 49-72.

[24] J. Ferec, E. Abisset-Chavanne, G. Ausias, F. Chinesta, On the use of interaction tensors to describe and predict rod interactions in rod suspensions, Rheol. Acta 53 (2014) 445-456.

[25] F. Folgar, Ch. Tucker, Orientation behavior of fibres in concentrated suspensions, J. Reinf. Plast. Comp. 3 (1984) 98-119.

[26] S. Jaganathana, H.V. Tafreshib, B. Pourdeyhimia, A realistic approach for modeling permeability of fibrous media: 3-d imaging coupled with CFD simulation, Chem. Eng. Sci. 63 (2008) 244-252.

[27] G.B. Jeffery, The motion of ellipsoidal particles immersed in a viscous fluid, Proc. R Soc. London A102 (1922) 161-179.

[28] M. Kroger, A. Ammar, F. Chinesta, Consistent closure schemes for statistical models of anisotropic fluids, J. Non-Newtonian Fluid Mech. 149 (2008) 40-55.

[29] H.L. Liu, W.R. Hwang, Permeability prediction of fibrous porous media with complex 3d architectures, Compos. Part A 43 (2012) 2030-2038.

[30] E. Lopez, E. Abisset-Chavanne, S. Comas-Cardona, C. Binetruy, F. Chinesta, Flow modeling of linear and nonlinear fluids in two and three scale fibrous fabrics, Int. J. Mater. Form. 9 (2016) 215-227.

[31] E. Lopez, A. Leygue, E. Abisset-Chavanne, S. Comas-Cardona, C. Aufrere, C. Binetruy, F. Chinesta, Flow modeling of linear and nonlinear fluids in two scale fibrous fabrics. advanced simulations, Int. J. Mater. Form. 10 (2017) 317-328.

[32] A. Ma, F. Chinesta, A. Ammar, M. Mackley, Rheological modelling of carbon nanotube aggregate suspensions, J. Rheol. (N Y N Y) 52/6 (2008) 1311-1330.

[33] A. Ma, F. Chinesta, M. Mackley, The rheology and modelling of chemically treated 
carbon nanotube suspensions, J Rheol. (N Y N Y) 53/3 (2009) 547-573.

[34] R. Mezher, et al., Direct simulation of concentrated fibre suspensions subjected to bending effects, Modelling Simul. Mater. Sci. Eng. 23 (2015) 055007.

[35] R. Mezher, M. Perez, A. Scheuer, E. Abisset-Chavanne, F. Chinesta, R. Keunings, Analysis of the folgar \& tucker model for concentrated fibre suspensions in unconfined and confined shear flows via direct numerical simulation, Composites Part A 91 (2016) 388-397.

[36] M. Perez, A. Scheuer, E. Abisset-Chavanne, F. Chinesta, R. Keunings, A multi-scale description of orientation in confined suspensions involving rods, J. Nonnewton Fluid Mech. 233 (2016) 61-74.

[37] M. Perez, Modelling and simulation of micro/nano structured fluids for engineered materials, ECN, 2016 Phd thesis dissertation.

[38] J. Phelps, Ch. Tucker, An anisotropic rotary diffusion model for fibre orientation in short and long fibre thermoplastics, J. Non-Newtonian Fluid Mech. 156/3 (2009) 165-176.

[39] E. Pruliere, A. Ammar, N.E. Kissi, F. Chinesta, Recirculating flows involving shor fibre suspensions: numerical difficulties and efficient advanced micro-macro solvers, Arch. Comput. Methods Eng., State of the Art Reviews 16 (2009) 1-30.

[40] S. Ranganathan, S.G. Advani, Fiber-fiber interactions in homogeneous flows of nondilute suspensions, J. Rheol. 35/ 8 (1991) 1499-1522.

[41] A. Scheuer, E. Abisset-Chavanne, F. Chinesta, R. Keunings, Second-gradient modelling of orientation development and rheology of confined suspensions, J. Non-
Newtonian Fluid Mech. 237 (2016) 54-64.

[42] S. Toll, Note: on the tube model for fiber suspensions, J. Rheol. 37/1 (1993) $123-125$.

[43] S. Toll, Packing mechanics of fiber reinforcements, Polym. Eng. Sci. 38/8 (1998) 1337-1350.

[44] S. Torquato, Statistical description of microstructures, Annu. Rev. Mater. Res. 32 (2002) 77-111.

[45] C.M. VanWyk, Note on the compressibility of wool, J Textile Inst. 37 (1946) T285-T292.

[46] B. Verleye, S.V. Lomov, A. Long, I. Verpoest, D. Roose, Permeability prediction for the meso-macro coupling in the simulation of the impregnation stage of resin transfer moulding, Compos. Part A 41 (2010) 29-35.

[47] J. Wang, C.A. Silva, J.C. Viana, F.W.J. van Hattum, A.M. Cunha, Ch. Tucker, Prediction of fibre orientation in a rotating compressing and expanding mold, Polym. Eng. Sci. (2008) 1405-1413.

[48] J. Wang, J. O'Gara, Ch. Tucker, An objective model for slow orientation kinetics in concentrated fibre suspensions: theory and rheological evidence, J. Rheol. (N Y N Y) 52/5 (2008) 1179-1200.

[49] C.C. Wong, A.C. Long, M. Sherburn, F. Robitaille, P. Harrison, C.D. Rudd, Comparisons of novel and efficient approaches for permeability prediction based on the fabric architecture, Compos. Part A 37 (2006) 847-857. 